\title{
Inverse C-arm Positioning for Interventional Procedures Using Real-Time Body Part Detection $^{\star}$
}

\author{
Christian Schaller, Christopher Rohkohl, Jochen Penne, Michael Stürmer, \\ and Joachim Hornegger \\ Chair of Pattern Recognition and Erlangen Graduate School in Advanced Optical \\ Technologies (SAOT), Friedrich-Alexander University Erlangen-Nuremberg, \\ Martensstr. 3, 91058 Erlangen, Germany \\ christian.schaller@informatik.uni-erlangen.de
}

\begin{abstract}
The automation and speedup of interventional therapy and diagnostic workflows is a crucial issue. One way to improve these workflows is to accelerate the image acquisition procedures by fully automating the patient setup. This paper describes a system that performs this task without the use of markers or other prior assumptions. It returns metric coordinates of the 3-D body shape in real-time for inverse positioning. This is achieved by the application of an emerging technology, called Time-of-Flight (ToF) sensor. A ToF sensor is a cost-efficient, offthe-shelf camera which provides more than 40,000 3-D points in realtime. The first contribution of this paper is the incorporation of this novel imaging technology ( $\mathrm{ToF}$ ) in interventional imaging. The second contribution is the ability of a C-arm system to position itself with respect to the patient prior to the acquisition. We are using the $3-\mathrm{D}$ surface information of the patient to partition the body into anatomical sections. This is achieved by a fast two-stage classification process. The system computes the ISO-center for each detected region. To verify our system we performed several tests on the ISO-center of the head. Firstly, the reproducibility of the head ISO-center computation was evaluated. We achieved an accuracy of (x: $1.73 \pm 1.11 \mathrm{~mm} / \mathrm{y}$ : $1.87 \pm 1.31 \mathrm{~mm} / \mathrm{z}: 2.91 \pm 2.62 \mathrm{~mm}$ ). Secondly, a C-arm head scan of a body phantom was setup. Our system automatically aligned the ISO-center of the head with the C-arm ISO-center. Here we achieved an accuracy of \pm $1 \mathrm{~cm}$, which is within the accuracy of the patient table control.
\end{abstract}

\section{Introduction and Related Work}

Workflow optimization is an important task in clinical procedures. The combination of increased life expectancy and advancements in the field of medicine

\footnotetext{
* The authors gratefully acknowledge funding of the International Max-PlanckResearch-School for Optics and Imaging (IMPRS-OI) and the Erlangen Graduate School in Advanced Optical Technologies (SAOT) by the German National Science Foundation (DFG) in the framework of the excellence initiative. We also thank the Siemens AG, Health Care Sector, Forchheim, Germany for supporting this work.
} 
have resulted in a constantly increasing number of patients. This in-turn creates a heavier workload for hospitals and healthcare systems in general. Thus, it is becoming progressively more important to develop efficient healthcare procedures without any decline on the quality of patient care. In the future, either healthcare costs will increase dramatically or smart cost-effective solutions for optimizing current workflows have to be found.

With that last goal in mind we examined the image acquisition workflows, in general, and analyzed them in terms of bottlenecks. In this paper we introduce a system to shorten the probably most important and for sure most time consuming part of this procedure: the initial patient positioning step. Image acquisition procedures have constantly been optimized within the past years. Today the scanning time for CT and C-arm systems is basically negligible. What is still very time consuming within the workflow of image acquisition procedures is the patient-dependent setup procedure. Each patient is physiologically relatively unique. There is also a diverse number of clinical procedures. As a result, the patient setup process cannot be easily generalized. Nevertheless, the correct positioning of the patient is a crucial parameter for the quality of image acquisition. Also for retrospective hybrid-image acquisitions it is important to acquire images at the correct position. Therefore, it is important that patient positioning is performed accurately, which can make it a very time consuming task. Especially in interventional procedures correct positioning of the image acquisition device is mandatory. Todays C-arm systems are already very intuitive to use. Strobel et al. 1] describe the setup procedure and the associated time consuming steps. Several fluoroscopic images have to be taken before the required body part is within the ISO-center of the C-arm. These steps are both very time consuming and applying additional radiation doses to the patient.

To automate and speed-up the setup procedure for interventional image acquisition procedures we suggest a system based on a ToF sensor. The proposed system partitions the whole body into several 3-D bounding boxes. It operates marker-lessly and does not rely on any other assumptions. The C-arm system receives exact metric coordinates from the proposed system which can then be used in automatically adjusting the C-arm's position with respect to the patient and for data acquisition. We call this inverse positioning, as the system is positioned with respect to the target and not the target with respect to the system. Furthermore, the C-arm knows exactly the bounds of the object to scan and an instantaneous collision detection can be provided. Last but not least, the proposed solution is very cost-effective.

The importance of a solution to these problems is also reflected by prior work. Grimson et al. 2] introduced a system wich supports optical tracking of patient and instrument locations using surface data. Navab et al. 3 proposed a system for intraoperative positioning and repositioning of mobile $\mathrm{C}$-arms using a cameraaugmented mobile C-arm. This system speeds up the whole procedure and also reduces the radiation of the patient as it decreases the number of images which have to be acquired for positioning the patient. Using an optical camera and $\mathrm{X}$-ray/optical markers the system supports guidance for C-arm repositioning. 


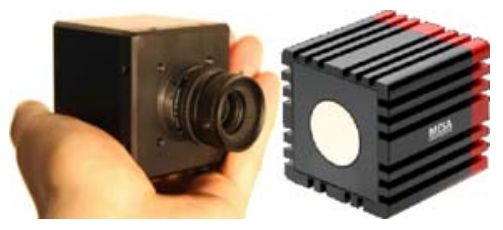

Fig. 1. Examples for ToF sensor models. On the left side: CamCube from PMDtec GmbH, Germany. On the right side: SR4000 from MESA Imaging, AG, Switzerland (images taken from manufactor websites)

C-arm systems also suffer from their narrow field-of-view. Wang et al. 4] suggest a stitching algorithm for scaning long bones using a C-arm system. They, too use an additional optical camera to augment different views. Ladikos et al. 5] provide a method for collision detection for a C-arm environment. An array of optical cameras is used to generate a 3-D representation of the operation room. All of these applications are either based on markers and/or use multiple cameras/systems. The proposed system is independent of markers and only needs a single sensor.

\section{Materials and Methods}

\subsection{Time-of-Flight Sensor}

ToF sensors provide a direct way to acquire 3 -D surface information [6]. ToF sensors have several advantages over other 3 -D surface acquisition techniques. The most promising advantage is that $\mathrm{ToF}$ is on its way to become a component of mass markets like consumer electronics and the automotive industry. Currently a high-end ToF sensor is available for about USD 7,000 (see Fig. 1). A target price for ToF sensors of a few hundred dollars can be expected in the near future. ToF sensors also render calibration steps, which are mandatory for stereo based systems, unnecessary. This is based on the monocular all-solid-state architecture of ToF sensors. Such an architecture also enables a high portability of the system and a variety of integration prospects in existing systems. Recent ToF sensors provide data rates up to 25 frames per second with an lateral resolution of up to $204 \times 204$ pixels. Each of these 3 -D points provides precise metric information in the sensor coordinate system. Recently several systems for medical applications like respiratory motion or patient positioning using a ToF sensor were proposed [78. Further information about the principle of ToF sensors can be found in Xu et al. 6]. A detailed discussion about the advantages of ToF sensors over other 3 -D surface acquisition techniques can be found in Schaller et. al [7].

\subsection{Body Part Detection}

In this section we will give an overview of how a ToF sensor enables inverse realtime positioning. We will introduce a generalized, very fast and fairly simple 


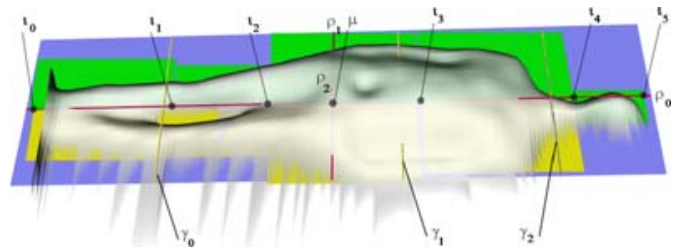

Fig. 2. Overview: The red lines indicate the three principal axes $\rho_{0}, \rho_{1}, \rho_{2}$. The three golden sections $\gamma_{0}, \gamma_{1}, \gamma_{2}$ are shown as yellow lines. Furthermore, all intersection points $\iota_{k}$ and the centroid $\mu$ are also depicted.

algorithm to solve this task. The proposed algorithm for body part detection consists of two main parts which require a preprocessing and segmentation of the ToF sensor data. The first part describes an effective and robust two-stage classification procedure. At the end of this first subtask, the 3-D body surface is subdivided into multiple anatomically meaningful regions of interest. The second part computes bounding boxes for each of these regions and their corresponding ISO-center. Furthermore, a fairly simple calibration method for aligning the computed ISO-center with the C-arm ISO-center is introduced.

The ToF sensor is rigidly mounted above the patient table and the whole patient is within the field of view of the ToF sensor. We denote $\mathbf{P}$ the $M \times N$ 3-D points of interest acquired by a ToF sensor.

$$
\mathbf{P}=\left[\mathbf{p}_{i, j}\right], i \in\{0,1, . ., M-1\}, j \in\{0,1, . . N-1\}
$$

Typically ToF data is affected by noise. To reduce this noise we apply both, a bilateral filter [9] and a temporal averaging filter. The averaging filter returns the average $3-\mathrm{D}$ point cloud using data from the last $n$ accumulated 3-D point clouds. To reduce the amount of data and to identify $3-\mathrm{D}$ points belonging to the body we detect the patient table and compute a virtual plane. For this task we use a Hough-Transform like method based on surface normals described in Schaller et al. [7. Knowing the virtual table plane we can discard all 3-D points which do not belong to the patient. As a result for further computations only relevant 3 -D points $\hat{\mathbf{P}} \subseteq \mathbf{P}$ ( $\subseteq$ denotes a subset of points) belonging to the body have to be considered. To speed-up the classification process, we apply a normalization on the remaining $3-D$ points $\hat{\mathbf{P}}$. Therefore a Karhunen-LoeveTransformation (PCA) is performed. As a result the origin of the coordinate system is placed in the centroid $\mu$ of the point cloud $\hat{\mathbf{P}}$ and the axes are aligned with the axial, sagittal and coronal plane of the patient. The main advantage we achieve is parallelism of the bounding boxes to the axes. This results in a more computationally efficient run-time for the algorithm. The three principal axes computed by the transformation are denoted as $\rho_{0}, \rho_{1}, \rho_{2}$ (see Fig. 2). We have prior knowledge about the shape of the human body. Therefore, we can assume that $\rho_{0}$ corresponds to the height of the patient, $\rho_{1}$ to the width and $\rho_{2}$ to the depth.

One can then compute five intersections $\left(\iota_{k}\right.$, where $\left.k \in\{0,1, . ., 5\}\right)$ along the first principle axis (see Fig. 2). The outer most intersection points $\iota_{0}$ and $\iota_{5}$ are 


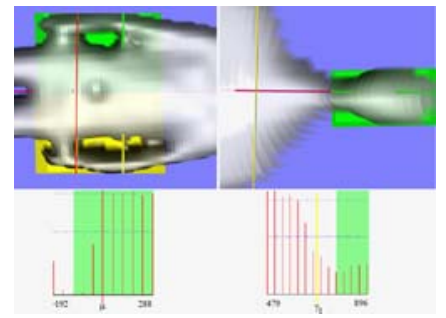

Fig. 3. Refinement of the bounding boxes. Left: Detection of the pelvic region with the corresponding histogram below. $\mu$ is the origin of the coordinate system. The search interval $d_{1}$ is $30 \mathrm{~cm}$ in each direction with a bining size of $4.8 \mathrm{~cm}$. Right: Detection of the neck with the corresponding histogram below. $\gamma_{1}$ is the upper golden section intersection. The search interval $d_{0}$ is $10 \mathrm{~cm}$ with a bining size of $3.2 \mathrm{~cm}$.

defined by the outermost $3-\mathrm{D}$ points on $\rho_{0}$. Again, we utilize prior knowledge about the shape of the human body. For a coarse first stage initialization of the classification we compute three golden intersections $\gamma_{0}, \gamma_{1}, \gamma_{2}$ along $\rho_{0}$ (see Fig. 2). The golden section $\gamma_{2}$ roughly indicates the position of the neck. $\gamma_{1}$ is located near the diaphragm and $\gamma_{0}$ at the knees of the person. These points constrain the search space for the exact position of the remaining intersection points.

To refine these coarse initial values we introduce a second stage in our classification. Two histograms $H_{0}$ and $H_{1}$ along $\rho_{0}$ are computed. The vertical axis of $H_{0}$ bins the silhouette of the body along the positive $p_{1}$ axis, while the vertical axis of $H_{1}$ bins it along the positive $p_{2}$ direction. The horizontal axis of $H_{0}$ ranges from $\gamma_{2}-d_{0}$ to $\gamma_{2}+d_{0}$. The horizontal axis of $H_{1}$ ranges from $\gamma-d_{1}$ to $\gamma+d_{1} \cdot d_{0}$ and $d_{1}$ are emirically determined. Based on these histograms, $\iota_{4}$ and $\iota_{5}$ are the minima of $H_{0}$ and $H_{1}$ accordingly. $\iota_{3}$ is then defined as the mid-point between $\iota_{4}$ and $\iota_{5}$, while $\iota_{1}$ is set to $\gamma_{0}$.

We can then use these intersections as an input for the second part of the algorithm. This part computes bounding boxes using $\iota_{0}, . ., \iota_{5}$. With the exception of the head, left and right bounding boxes are computed for each body segment (e.g. left and right abdomen), where $\rho_{0}$ acts as a delimiter. In addition to the boundary values, the ISO-center and the volume is computed for each box. Figure 4 shows the full body part segmentation.

Before we are able to position a target, a fairly simple calibration step has to be performed. The following has to be done only once for the whole system. A coin is placed on a box on the patient table (see Fig. (5). The ISO-center $I_{C a r m}$ of the $\mathrm{C}$-arm is manually aligned with the center of the coin. We use two fluoroscopic images, one from $0^{\circ}$ and one from $90^{\circ}$ to do this. After determining $I_{C a r m}$, the corresponding 3 -D coordinate (ISO-center) of the center of the coin $I_{T o F}$ in the ToF coordinate system has to be identified. These two points, $I_{C a r m}$ and $I_{T o F}$ can be considered as the origins of each of the corresponding coordinate systems. To position a bounding box ISO-center, the 3-D coordinate of the ISO-center is shifted to $I_{T o F}$. 


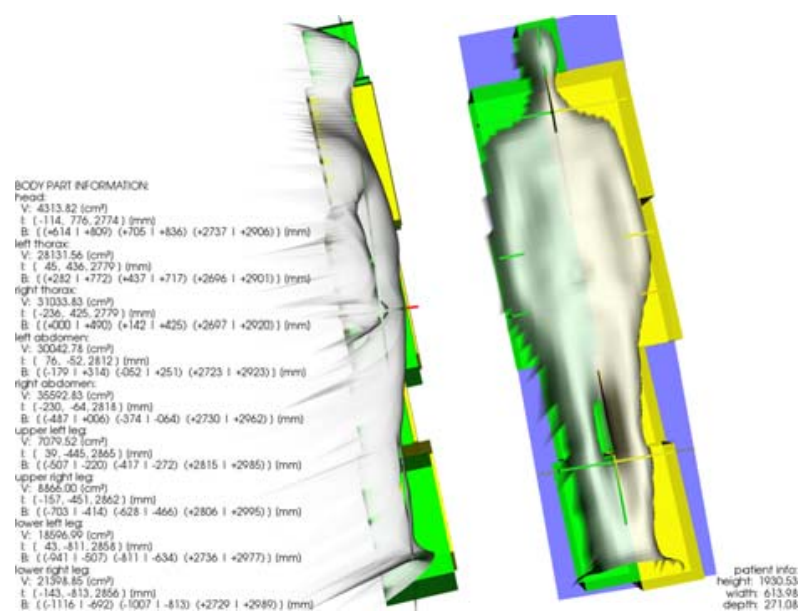

Fig. 4. Full body acquisition using a ToF sensor including bounding boxes (side view and top view). On the left side information about the different bounding boxes is shown. $\mathrm{V}$ shows the volume of the bounding box in $\mathrm{cm}^{3}$, I the ISO-center of the bounding box in $\mathrm{mm}$ and $\mathrm{B}$ the bounds of the actual bounding box in $\mathrm{mm}$. On the lower right side, some basic information about the dimensions of the patient is displayed.

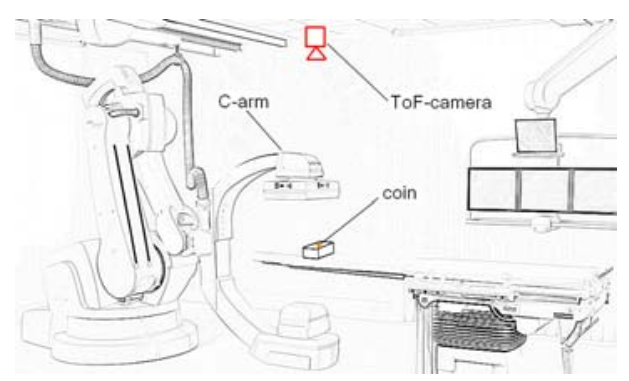

Fig. 5. Coin calibration: A coin is placed on a box on the patient table. The coin is aligned in the ISO-center of the C-arm. Afterwards, the corresponding 3-D coordinate of the position of the coin in the ToF point cloud can be determined.

\section{$3 \quad$ Experiments and Results}

For the evaluation we used a SR-3000 ToF sensor from MESA Imaging, AG, Switzerland and a C-arm system of the Artis zee family from Siemens AG, Healthcare Sector, Germany. We rigidly mounted the ToF sensor on the ceiling above the patient table so that the patient can be fully visible. The ToF sensor has a resolution of $176 \times 144$ pixels with a field of view of $47.5^{\circ}$ and $39.6^{\circ}$ respectively. The depth accuracy after preprocessing the data is below $1 \mathrm{~mm}$ per pixel.

We first examined the reproducibility of the ISO-center computation. Without loss of generality, this was done for the head ISO-center. For the other 


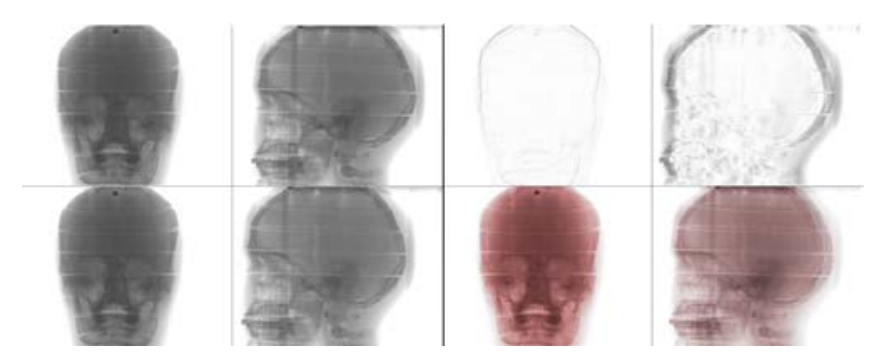

Fig. 6. Phantom evaluation: Two upper left images: $0^{\circ}$ and $90^{\circ}$ projections of manually aligned head (gold standard). Two lower left images: $0^{\circ}$ and $90^{\circ}$ projections of automatically aligned head using the proposed method. Two upper right images: $0^{\circ}$ and $90^{\circ}$ projection difference images of manually and automatically aligned head. Two lower right images: $0^{\circ}$ and $90^{\circ}$ projections of manually and automatically aligned head (automatically aligned projections are superimposed in red).

ISO-centers this could be done in a similar manner. The head ISO-center of three persons was computed 70 times per person. We took the mean $\mathrm{x}-\mathrm{y}$ - $\mathrm{z}$ - coordinate as our reference ISO-center. We again computed 140 head ISO-centers for each of these persons while they were in the same position. The mean squared errors between those measurments and the reference in $\mathrm{x}-\mathrm{y}$ - $\mathrm{z}$ - direction (corresponding to $\left.\rho_{0}, \rho_{1}, \rho_{2}\right)$ are: x: $1.73 \pm 1.11 \mathrm{~mm}, \mathrm{y}: 1.87 \pm 1.31 \mathrm{~mm}, \mathrm{z}: 2.91 \pm 2.62 \mathrm{~mm}$. Furthermore, we computed the inter-subject standard deviation on these datasets: $\mathrm{x}$ : $3.16 \mathrm{~mm}$ y: $2.85 \mathrm{~mm}$ z: $4.42 \mathrm{~mm}$. To evaluate our approach within a clinical setup a body phantom was placed in a $\mathrm{C}$-arm environment. We were again interested in the ISO-center of the phantom head. The phantom was shifted in all three room dimensions multiple times. After computing the ISO-center $I_{h}$ of the head bounding box with respect to the previously computed $I_{T o F}$ we could directly compute the translation to align $I_{h}$ with $I_{\text {Carm }}$. A gold standard was defined by manually aligning the ISO-center of the head using the same technique as for the previously described coin calibration (see Fig. 6). This is also the way it is done in hospitals today. We compared the table position of the gold standard alignment and the automatic alignment. In all cases we were able to automatically position the head in the $\mathrm{x}$ and the $\mathrm{y}$ direction within the accuracy of the patient table. According to the manufactor the accuracy is $1 \mathrm{~cm}$. In the z-direction, we had an error of $\pm 1 \mathrm{~cm}$ (see Fig. 6) because the z-coordinate of the ISO-center heavily depends on the quality of the table segmentation. On a standard CPU (2.0 GHz dual-core) our algorithm has an execution time of $65 \mathrm{~ms}$. Including data acquisition, preprocessing and segmentation, the overall execution time is about $143 \mathrm{~ms}$.

\section{Conclusion}

Automation of time consuming steps is a key competency of future clinical procedures and workflow aware hospitals. We have shown that our system for fully 
automatic patient setup is able to identify anatomical regions for image acquisition in real-time. This information can be used to align the ISO-center of anatomical targets with the ISO-center of a C-arm system fully automatically. For this task, a 3-D surface acquired by a ToF sensor is analyzed. A two-stage classification process is introduced to identify body parts and to compute the corresponding ISO-centers. The extracted information can be directly used to control any image acquisition devices. This results in an inverse positioning, where the image acquisition device can be positioned according to the patient. Furthermore, several patient dependent metric information are provided by the system. This information can be used for e.g., initial positioning, collision detection, dose estimation or respiratory motion correction [7.

\section{References}

1. Strobel, N., Meissner, O., Boese, J., Brunner, T., Heigl, B., Hoheisel, M., Lauritsch, G., Nagel, M., Pfister, M., Rúhrnschopf, E.-P., Scholz, B., Schreiber, B., Spahn, M., Zellerhoff, M., Klingenbeck-Regn, K.: 3D Imaging with Flat-Detector C-Arm Systems, 3rd edn., pp. 33-51. Springer, Heidelberg (2009)

2. Grimson, W.E.L., Leventon, M.E., Ettinger, G., Chabrerie, A., Ozlen, F., Nakajima, S., Atsumi, H., Kikinis, R., Black, P.: Clinical experience with a high precision imageguided neurosurgery system. In: Wells, W.M., Colchester, A.C.F., Delp, S.L. (eds.) MICCAI 1998. LNCS, vol. 1496, pp. 63-73. Springer, Heidelberg (1998)

3. Navab, N., Wiesner, S., Benhimane, S., Euler, E., Heining, S.M.: Visual servoing for intraoperative positioning and repositioning of mobile C-arms. In: Larsen, R., Nielsen, M., Sporring, J. (eds.) MICCAI 2006. LNCS, vol. 4190, pp. 551-560. Springer, Heidelberg (2006)

4. Wang, L., Traub, J., Heining, S.M., Benhimane, S., Euler, E., Graumann, R., Navab, N.: Long bone X-ray image stitching using camera augmented mobile C-arm. In: Metaxas, D., Axel, L., Fichtinger, G., Székely, G. (eds.) MICCAI 2008, Part II. LNCS, vol. 5242, pp. 578-586. Springer, Heidelberg (2008)

5. Ladikos, A., Benhimane, S., Navab, N.: Real-time 3D reconstruction for collision avoidance in interventional environments. In: Metaxas, D., Axel, L., Fichtinger, G., Székely, G. (eds.) MICCAI 2008, Part II. LNCS, vol. 5242, pp. 526-534. Springer, Heidelberg (2008)

6. Xu, Z., Schwarte, R., Heinol, H., Buxbaum, B., Ringbeck, T.: Smart Pixel - Photometric Mixer Device (PMD) / New System Concept of a 3D-Imaging-on-a-Chip. In: 5th International Conference on Mechatronics and Machine Vision in Practice, pp. 259-264 (1998)

7. Schaller, C., Penne, J., Hornegger, J.: Time-of-Flight Sensor for Respiratory Motion Gating. Medical Physics 35(7), 3090-3093 (2008)

8. Schaller, C., Adelt, A., Penne, J., Hornegger, J.: Time-of-flight sensor for patient positioning. In: Proceedings of SPIE Medical Imaging 2009: Visualization, Imageguided Procedures and Modeling (2009)

9. Paris, S., Durand, F.: A fast approximation of the bilateral filter using a signal processing approach. Int. J. Comput. Vision 81(1), 24-52 (2009) 